\title{
Scanning Probe Microscopy of Crosslinked Proteins: from Textiles to Rubbers
}

\author{
Mickey Huson and Jane Maxwell
}

CSIRO Textile and Fibre Technology, PO Box 21, Belmont, Victoria 3216, Australia

The Scanning Probe Microscope (SPM) is a versatile tool which not only records images of samples but can also be used to gather information about the nano-mechanical properties (stiffness, hardness, adhesion, visco-elasticity and friction) of the surface of a material [1]. When used in Electric Force Mode (EFM) it can even determine electrical properties. The SPM can operate in a gaseous or liquid environment, making it ideal for investigating a wide range of polymeric and biological materials.

CSIRO Textile and Fibre Technology was, for nearly 50 years, a Division who's primary interest was wool, thus within the Division there is a lot of expertise on wool, a crosslinked keratin protein. In recent years, as part of a diversification strategy, we have extended our interests to look at other crosslinked proteins such as gelatin and resilin. This presentation reports on a range of applications where an SPM has been usefully employed to investigate these materials.

Imaging the surface of a wool fibre can give immediate information about damage or contamination, morphology and scale height or roughness (Fig. 1). Scale height is of particular interest as the scales are implicated in the propensity of wool to felt when fibres or fabric undergo mechanical agitation. Felting is particularly severe when the agitation takes place in water and the SPM's ability to image in water has been utilized to show that, on average, the scale height increases $21 \%$ on going from dry to fully immersed in water [2] and that the scales are much softer when wet [3]. The combination of increased scale height and softer surfaces results in increased differential friction between fibres leading to greater entanglement and hence felting. The surface of wool fibres have also been compared to model substrates using SPM adhesion studies (Fig. 2) and shown to vary depending on the environment. The results suggest that the $\mathrm{C} 21$ lipid outer layer (18 methyleicosanoic acid) present in ambient air reorients itself upon exposure to an aqueous environment so that the protein (amide) groups of the proteolipid are oriented outwards. This supports the notion of a dynamic fibre surface, capable of altering its structure in response to different environments (Fig. 2) [4]. Other ongoing work using friction measurements and chemical mapping to follow surface modification will also be reported. If the fibre is embedded and then sectioned the internal structure becomes visible and information can be obtained about the size and shape of the different cells and the cell membrane complex (Fig. 1). EFM has been used on fibre surfaces and cross-sections to track nanogold labels, used in surface reactivity studies, and also intrinsically conductive polymers applied to wool with the aim of producing conductive fabrics.

Gelatin is partly denatured collagen, which at room temperature forms a 3 dimensional rubber-like network which is capable of swelling in water. The ability to control the degree of swelling and extent of crosslinking are one of the reasons that gelatin remains pre-eminent in photographic film manufacturing. SPM force curves have been used to examine the homogeneity of thin films of gelatin since variations in crosslink density lead to variations in hardness.

The protein resilin, found in most if not all insects, is an elastic energy storage protein which facilitates flight (in flies, bees and dragonflies), enables sound production (in cicadas) and allows 
fleas and spittle bugs to achieve their remarkable jumping feats. A CSIRO team have recently produced a recombinant form of resilin protein with rubber-like characteristics [5] and, in a novel use of SPM [6], resilience has been measured and both the natural and the recombinant protein shown to have resilience of better than $90 \%$.

\section{References}

1. S. Magonov and D.A. Chernoff, Atomic Force Microscopy, in "Comprehensive Desk Reference of Polymer Characterization and Analysis", Ed. R.F. Brady, Jr., Oxford University Press, Washington, 2003.

2. T.L. Phillips et al., Text. Res. J. 65 (1995) 445.

3. J.M. Maxwell and M.G. Huson, Rev. Sci. Instr. 73 (2002) 3520.

4. J.M. Maxwell and M.G. Huson, Micron 36 (2005) 127.

5. C.M. Elvin et al., in preparation.

6. M.G. Huson and J.M. Maxwell, in preparation.

7. This work was supported in part by Australian Wool Innovation Ltd. and a CSIRO Nanotechnology Emerging Sciences Initiative grant.

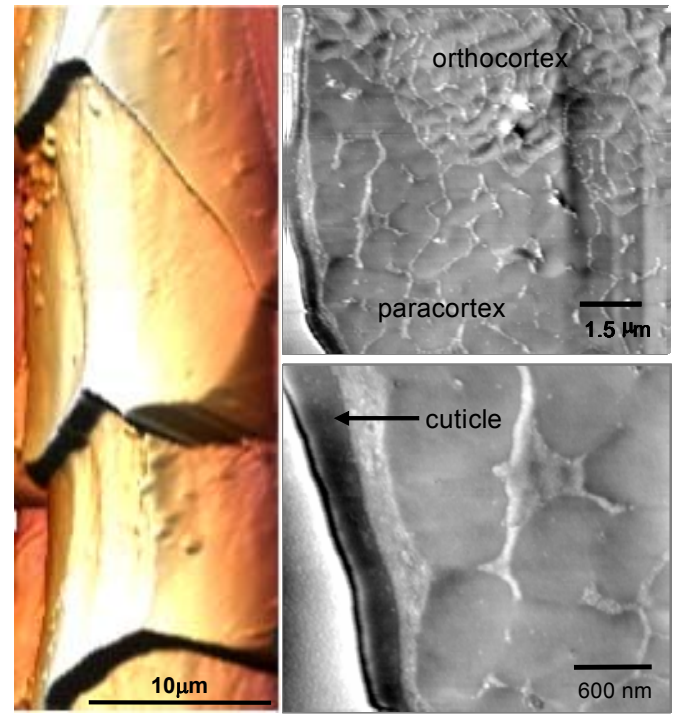

FIG. 1. Wool fibre showing surface cuticle cells in water (left, height range $4 \mu \mathrm{m}$ ) and cross- sections showing internal structure (right).

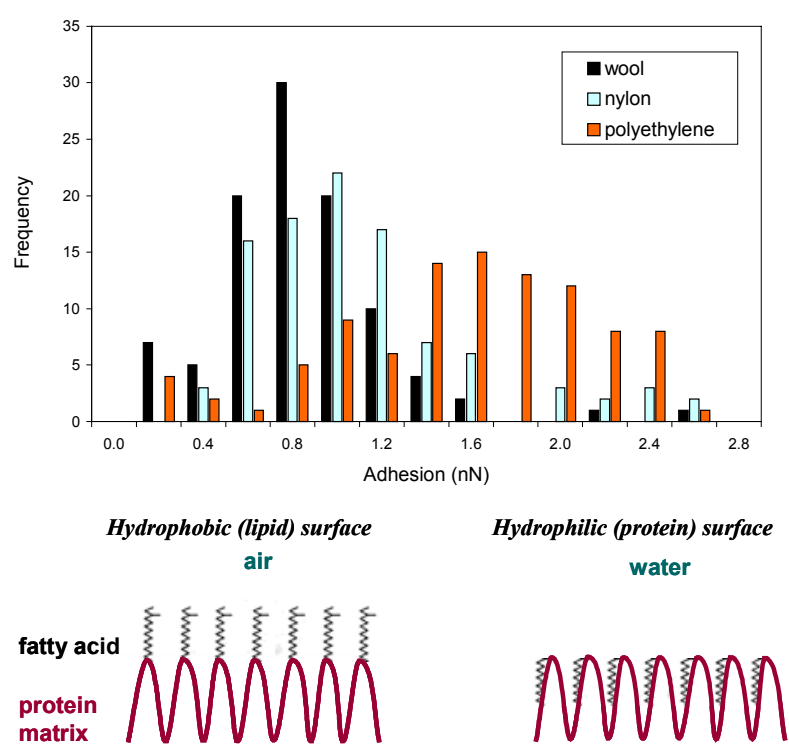

FIG. 2. Adhesion in water, (above) between a standard $\mathrm{Si}_{3} \mathrm{~N}_{4}$ tip and wool, nylon and polyethylene and (below) a schematic diagram depicting a proposed model of the surface of a wool fibre in air and in water. 